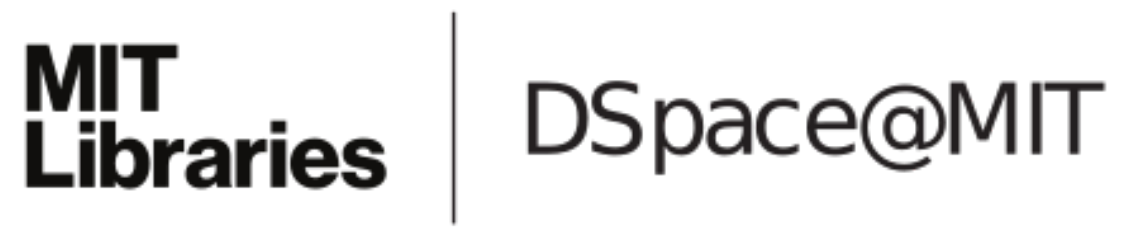

\author{
MIT Open Access Articles
}

Laboratory investigation of lateral dispersion within dense arrays of randomly distributed cylinders at transitional Reynolds number

The MIT Faculty has made this article openly available. Please share how this access benefits you. Your story matters.

Citation: Tanino, Yukie, and Heidi M. Nepf. "Laboratory investigation of lateral dispersion within dense arrays of randomly distributed cylinders at transitional Reynolds number." Physics of Fluids 21.4 (2009): 046603-10. (C) 2009 American Institute of Physics.

As Published: http://dx.doi.org/10.1063/1.3119862

Publisher: American Institute of Physics

Persistent URL: http://hdl.handle.net/1721.1/60994

Version: Final published version: final published article, as it appeared in a journal, conference proceedings, or other formally published context

Terms of Use: Article is made available in accordance with the publisher's policy and may be subject to US copyright law. Please refer to the publisher's site for terms of use. 


\title{
Laboratory investigation of lateral dispersion within dense arrays of randomly distributed cylinders at transitional Reynolds number
}

\author{
Yukie Tanino ${ }^{\text {a) }}$ and Heidi M. Nepf ${ }^{\text {b) }}$ \\ Massachusetts Institute of Technology, 77 Massachusetts Avenue, Cambridge, Massachusetts 02139, USA
}

(Received 24 June 2008; accepted 24 March 2009; published online 28 April 2009)

\begin{abstract}
Relative (effective) lateral dispersion of a passive solute was examined at transitional Reynolds numbers within a two-dimensional array of randomly distributed circular cylinders of uniform diameter $d$. The present work focuses on dense arrays, for which previously developed theory [Y. Tanino and H. M. Nepf, J. Fluid Mech. 600, 339 (2008)] implies that the asymptotic (long-time/ long-distance) dispersion coefficient, when normalized by the mean interstitial fluid velocity, $\langle\bar{u}\rangle$, and $d$, will only exhibit a weak dependence on Reynolds number, $\operatorname{Re}_{d} \equiv\langle\bar{u}\rangle d / \nu$, where $\nu$ is the kinematic viscosity. However, the advective distance required to reach asymptotic dispersion is expected to be controlled by pore-scale mixing, which is strongly $\mathrm{Re}_{d}$-dependent prior to the onset of full turbulence. Laser-induced fluorescence was used to measure the time-averaged lateral concentration profiles of solute released continuously from a point source in arrays of solid volume fraction $\phi=0.20$ and 0.35 at $\operatorname{Re}_{d}=48-120$. Results are compared to previous measurements at higher $\mathrm{Re}_{d}$. Lateral dispersion reaches the same rate as asymptotic dispersion in fully turbulent flow at $x \approx 154 d$ at $\left(\phi, \operatorname{Re}_{d}\right)=(0.20,110-120)$ and at $x \approx 87 d$ at $\left(\phi, \operatorname{Re}_{d}\right)=(0.35,300-390)$. In contrast, dispersion does not reach the fully turbulent flow limit at $\operatorname{Re}_{d}<100$ within the range of $x$ considered. Also, concentration profiles deviate further from a Gaussian distribution at $\phi=0.35$ than at 0.20 for similar $\operatorname{Re}_{d}$ and $x \phi / d$. From these observations, it can be inferred that the pre-asymptotic regime extends farther downstream, in terms of the number of cylinders spanned, at lower $\operatorname{Re}_{d}$ and at larger $\phi$. (C) 2009 American Institute of Physics. [DOI: 10.1063/1.3119862]
\end{abstract}

\section{INTRODUCTION}

Obstructed flows, i.e., flows through an array of solid bodies, are ubiquitous in the environment. Classic examples include groundwater flow and flow around buildings, trees, and aquatic plants. The simplest obstructed flow is uniform flow through a two-dimensional array of circular cylinders. Such an array is often used as an idealized porous medium to better understand how obstructions affect flow and transport (e.g., Refs. 1-3). In addition, certain emergent aquatic plants, which are the application that motivates this study, have stems that are approximately cylindrical, and quantitative measurements obtained in cylinder arrays can be applied directly to these systems.

In this paper, we consider solute transport in a homogeneous, two-dimensional array of rigid circular cylinders of uniform diameter $d$ distributed randomly with a constant density $m$ (cylinders per unit horizontal area). The corresponding solid volume fraction is $\phi=m \pi d^{2} / 4$. The center-tocenter distance from a particular cylinder to its nearest neighbor is denoted by $s_{n c}$ (Fig. 1). The corresponding distance between the surfaces of the cylinders is denoted by $s_{n}\left(=s_{n c}-d\right)$. In this paper, we use $d$ and $\left\langle s_{n}\right\rangle_{A}$, the mean nearest-neighbor separation defined between cylinder surfaces, as the characteristic geometric scales of the array. Here, \langle\rangle$_{A}$ denotes an average over many cylinders in the

\footnotetext{
a) Author to whom correspondence should be addressed. Electronic mail: ytanino@alum.mit.edu.

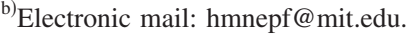

array. This choice of length scales is motivated by the previous ${ }^{4}$ observation that the square root of the permeability and the integral length scale of turbulence are approximately equal to $\left\langle s_{n}\right\rangle_{A}$ for a wide range of $\phi(\phi<0.25$ and 0.091 $\leq \phi \leq 0.35$, respectively), and by the availability of an analytical solution for $\left\langle s_{n}\right\rangle_{A}$ that is a function only of $d$ and $\phi$.

The Cartesian coordinates $\boldsymbol{x}=(x, y)$ are defined such that the $x$-axis is aligned with the fluid velocity averaged over time and the fluid volume, denoted by $\langle\bar{u}\rangle$, and the $y$-axis is in the horizontal plane and perpendicular to the $x$-axis (Fig. 1). The overbar denotes an average over a time interval much longer than those associated with turbulent fluctuations and vortex shedding. \langle\rangle denotes an average over infinitesimally thin fluid volume that span many cylinders or, equivalently, an ensemble average, i.e., an average over many arrays with the same $\phi, d$, and flow conditions, but with different cylinder configurations. We only consider constant, uniform mean flow, i.e., time-independent $\langle\bar{u}\rangle$.

The two length scales identified above can be used to define two Reynolds numbers, $\operatorname{Re}_{d} \equiv\langle\bar{u}\rangle d / \nu$ and $\operatorname{Re}_{s} \equiv\langle\bar{u}\rangle\left\langle s_{n}\right\rangle_{A} / \nu$, where $\nu$ is the kinematic viscosity. It is well established that flow patterns around isolated cylinders are similar at the same $\mathrm{Re}_{d}$. Detailed descriptions of these flows are available in classic papers and in standard fluid mechanics text books, e.g., Refs. 5-7, so only the key regimes relevant to this paper are identified here. As $\operatorname{Re}_{d}$ is increased from Stokes flow, flow remains steady and laminar everywhere in the fluid up to $\operatorname{Re}_{d} \approx 40$. At $\operatorname{Re}_{d} \approx 40$, the isolated cylinder wake begins to oscillate periodically. Next, at 


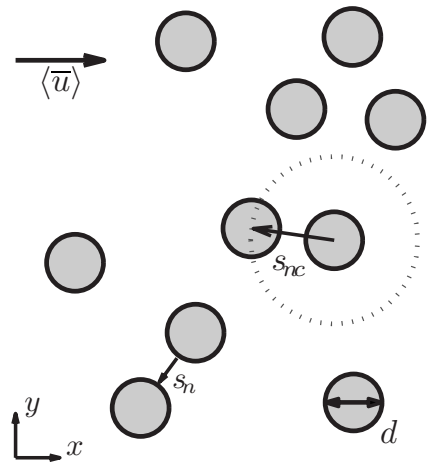

FIG. 1. Key parameters for a two-dimensional array of cylinders of uniform diameter $d$. In a random array, the center-to-center distance to the nearest neighbor, $s_{n c}$, differs for each cylinder.

$\mathrm{Re}_{d} \approx 90$, vortices begin to periodically shed from the cylinder. This unsteady, laminar wake regime continues until $\operatorname{Re}_{d} \approx 200$, beyond which the periodic motion of the wake gradually breaks down and the wake becomes turbulent as $\operatorname{Re}_{d}$ is increased further. The wake becomes fully turbulent at roughly $\mathrm{Re}_{d} \approx 5000$. $^{6}$

At sufficiently small $\phi$, flow around each cylinder in the array resembles flow past an isolated cylinder. In this limit, where $\left\langle s_{n}\right\rangle_{A}$ tends to infinity, $\operatorname{Re}_{d}$ is the relevant Reynolds number and, for example, transitions between the flow regimes described above occur at the same $\mathrm{Re}_{d}$ as in the isolated cylinder wake. At sufficiently large $\phi$, the array resembles a network of intersecting channels of width $\left\langle s_{n}\right\rangle_{A}$. Accordingly, flow in such arrays is expected to be kinematically and dynamically similar at the same $\mathrm{Re}_{s}$. Arrays considered in this study fall between these limits, such that neither $\operatorname{Re}_{d}$ or $\operatorname{Re}_{s}$ captures the similarity across flows in different $\phi$. For simplicity, results will be discussed in terms of $\mathrm{Re}_{d}$ only. In the laboratory experiments considered in the present paper, $d$ was kept constant, and differences in $\operatorname{Re}_{d}$ result entirely from differences in $\langle\bar{u}\rangle / \nu$, i.e., $\operatorname{Re}_{d}$ dependence refers specifically to the dependence on $\langle\bar{u}\rangle / \nu$.

It is convenient to describe the conservation of a passive solute in a two-dimensional array in terms of temporally and spatially averaged parameters, ${ }^{8}$

$$
\begin{aligned}
\frac{\partial\langle\bar{c}\rangle}{\partial t} & +\left\langle\bar{v}_{j}\right\rangle \frac{\partial\langle\bar{c}\rangle}{\partial x_{j}} \\
& =-\frac{\partial}{\partial x_{j}}\left\{\left\langle\overline{v_{j}^{\prime} c^{\prime}}\right\rangle+\left\langle\bar{v}_{j}^{\prime \prime} \vec{c}^{\prime \prime}\right\rangle-D_{m}\left\langle\frac{\partial}{\partial x_{j}}\left(\langle\bar{c}\rangle+\bar{c}^{\prime \prime}\right)\right\rangle\right\} .
\end{aligned}
$$

Here, ${ }^{-\prime}$ denotes the spatial fluctuations of the local temporal average (denoted by ${ }^{-}$), ' denotes the temporal fluctuations, $t$ is time, $c(\boldsymbol{x}, t)=\langle\bar{c}\rangle(\boldsymbol{x}, t)+\vec{c}^{\prime \prime}(\boldsymbol{x}, t)+c^{\prime}(\boldsymbol{x}, t)$ is the solute concentration, $\boldsymbol{v}(\boldsymbol{x}, t)=(u, v)=\langle\overline{\boldsymbol{v}}\rangle(\boldsymbol{x}, t)+\overline{\boldsymbol{v}}^{\prime \prime}(\boldsymbol{x}, t)+\boldsymbol{v}^{\prime}(\boldsymbol{x}, t)$ is the fluid velocity, and $D_{m}$ is the molecular diffusion coefficient of the solute. By definition, $\overline{c^{\prime}}, \overline{\boldsymbol{v}^{\prime}},\left\langle\vec{c}^{\prime \prime}\right\rangle,\left\langle\overline{\boldsymbol{v}}^{\prime \prime}\right\rangle=0$. Also, by our definition of the Cartesian coordinates, $\langle\bar{v}\rangle=0$. In uniform, time-independent mean flow through a spatiallyhomogeneous random array, long-range velocity correlations are not expected because of the obstructions. ${ }^{9,10}$ Accordingly,
Fickian dispersion is expected after sufficiently long time after the solute is introduced to the flow, once the spatial scale over which the mean concentration gradient varies exceeds the finite scales of velocity correlations. ${ }^{11}$ Previous ${ }^{3,4,12}$ and present (Sec. III C) observations of dispersion support this conjecture. Then, Eq. (1) reduces to

$$
\frac{\partial\langle\bar{c}\rangle}{\partial t}+\left\langle\bar{v}_{j}\right\rangle \frac{\partial\langle\bar{c}\rangle}{\partial x_{j}}=-\frac{\partial}{\partial x_{j}}\left(-K_{j j} \frac{\partial\langle\bar{c}\rangle}{\partial x_{j}}\right)=K_{j j} \frac{\partial^{2}\langle\bar{c}\rangle}{\partial x_{j}^{2}},
$$

where $K_{j j}$ are the coefficients for asymptotic (long-time/ long-distance) net dispersion. The first equality in Eq. (2) states that the sum of the fluxes on the right-hand side of Eq. (1) obeys Fick's law. The second equality states that $K_{j j}$ is spatially homogeneous, which is expected in a homogeneous array.

In the present study, we are only concerned with the lateral component of the macroscopic dispersion coefficient, $K_{y y}$. Consider an experiment in which solute particles are released continuously from a point source $(\boldsymbol{x}=\mathbf{0})$. Then, $\bar{c}(\boldsymbol{x})$ is the temporal average of the solute concentration observed at some point $\boldsymbol{x}$ during a single experiment, and $\langle\bar{c}\rangle(\boldsymbol{x})$ is its ensemble average. If the solute is transported according to Eq. (2), the lateral variance of its distribution is related to $K_{y y}$ as $^{13}$

$$
\frac{K_{y y}}{\langle\bar{u}\rangle d}=\frac{1}{2 d} \frac{d}{d x}\left[\left\langle\sigma_{y}^{2}(x)\right\rangle+\left\langle\left(\frac{m_{1}}{m_{0}}(x)\right)^{2}-\left\langle\frac{m_{1}}{m_{0}}(x)\right\rangle^{2}\right\rangle\right],
$$

where $\sigma_{y}^{2}$ and $m_{1} / m_{0}$ are the variance and the center of mass, respectively, of the time-averaged lateral concentration distribution at a given $x$ in a single experiment; $\left\langle m_{1} / m_{0}\right\rangle$ and the expression inside [ ] are the center of mass and the variance, respectively, of $\langle\bar{c}\rangle$ at a given $x$. From Eq. (2), it follows that $d\left\langle m_{1} / m_{0}\right\rangle / d x=0$. Further, since $m_{1} / m_{0}$ is the mean lateral displacement of many solute particles, $d\left\langle\left(m_{1} / m_{0}\right)^{2}\right\rangle / d x$ also approaches zero at sufficiently large $x$. Then, Eq. (3) reduces to

$$
\frac{K_{y y}}{\langle\bar{u}\rangle d} \approx \frac{1}{2 d} \frac{d}{d x}\left\langle\sigma_{y}^{2}(x)\right\rangle .
$$

The right-hand side of Eq. (4) is commonly referred to as the "relative" 14 or "effective",15 dispersion coefficient. Previous ${ }^{4,16}$ studies observed two properties at sufficiently high $\operatorname{Re}_{d}$ over the range of $x$ considered $\left(\operatorname{Re}_{d}>67-320\right.$ and $x / d>7-80$, depending on $\phi)$ : (i) $d\left\langle\sigma_{y}^{2}\right\rangle / d x$ remained constant in $x$, consistent with a spatially homogeneous $K_{y y} /(\langle\bar{u}\rangle d)$, and (ii) $d\left\langle\sigma_{y}^{2}\right\rangle / d x$ was independent of $\operatorname{Re}_{d}$. As a result, the corresponding $K_{y y} /(\langle\bar{u}\rangle d)$ was a function only of $\phi$ ( $d$ was kept constant).

Tanino and $\mathrm{Nepf}^{4}$ showed that $K_{y y} /(\langle\bar{u}\rangle d)$ in this high- $\mathrm{Re}_{d}$ regime can be described by the linear superposition of a model for turbulent diffusion and existing models for asymptotic (long-distance) dispersion associated with the tortuous flow path that fluid is forced to follow around the cylinders. In predicting the contribution from turbulent diffusion, fully turbulent flow, defined here to refer to flow that has achieved the maximum (and therefore $\mathrm{Re}_{d}$-independent) 


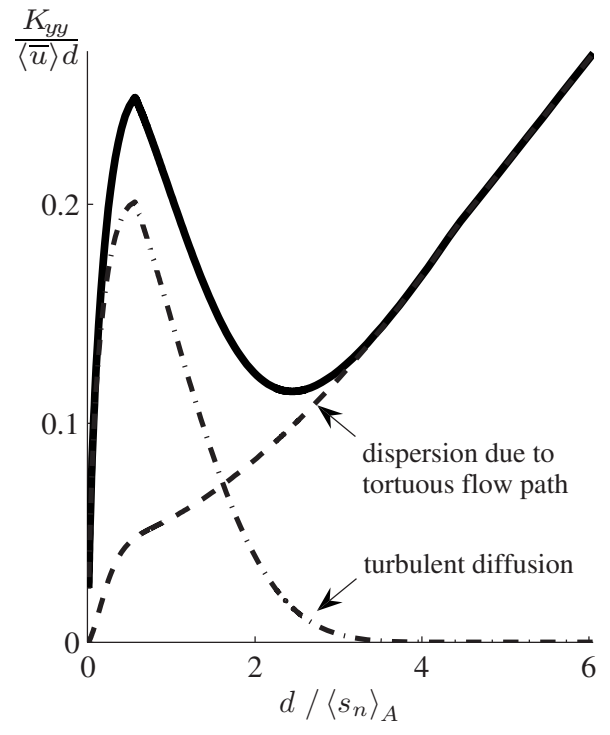

FIG. 2. $K_{y y} /(\langle\bar{u}\rangle d)$ for fully turbulent flow as predicted by the model of Tanino and Nepf (Ref. 4), with scaling constants determined therein and $d /\left\langle s_{n}\right\rangle_{A}$ as described by Eq. (A16) in Ref. 4. Dashed and dashed-dotted lines represent the contribution from the tortuous flow path and turbulent diffusion, respectively. Note that $d /\left\langle s_{n}\right\rangle_{A}$ increases monotonically with $\phi$.

mean turbulence intensity for that $\phi$, was assumed. Their model predicts that turbulent diffusion makes a nonnegligible (specifically, $\geq 10 \%$ ) contribution to $K_{y y} /(\langle\bar{u}\rangle d)$ at $d /\left\langle s_{n}\right\rangle_{A} \leq 2.6(\phi \leq 0.19)$ in fully turbulent flow (Fig. 2). Since turbulent diffusion must decrease as $\mathrm{Re}_{d}$ decreases below the fully turbulent regime, the theory implies that $K_{y y} /(\langle\bar{u}\rangle d)$ will also decrease as $\operatorname{Re}_{d}$ decreases in this $\phi$ range in turbulent flow. However, in unsteady laminar flow, periodic wakes may introduce a different mechanism of dispersion.

Conversely, the model predicts that at large $\phi(>0.19)$, turbulence does not contribute significantly to asymptotic dispersion and, consequently, that the contribution from the tortuous flow path, i.e., the time-averaged, spatially heterogeneous velocity field, dominates. In dense arrays, physical reasoning suggests that the time-averaged velocity field, and therefore its contribution to lateral dispersion, are determined primarily by the local cylinder configuration and, therefore, do not depend strongly on $\operatorname{Re}_{d}$. Therefore, $K_{y y} /(\langle\bar{u}\rangle d)$ at $\phi>0.19$ is not expected to depend strongly on $\mathrm{Re}_{d}$ either. To our knowledge, the $\mathrm{Re}_{d}$-dependence of the time-averaged velocity field has not been investigated directly. Nevertheless, the good agreement between experiment and the model of Tanino and Nepf, ${ }^{4}$ which assumes a $\mathrm{Re}_{d}$-independent contribution from the tortuous flow path, supports this conjecture. Indeed, Hill et al. ${ }^{17}$ made the same conjecture for dense arrays of randomly distributed spheres, based on their observation that the spatial variance of the transverse components of velocity normalized by $\langle\bar{u}\rangle^{2}$ differs by less than $4 \%$ between Stokes flow and $\operatorname{Re}_{d}=113$ in their numerical simulations at $\phi=0.588$. The same conjecture has also been made for rhombohedrally distributed spheres. ${ }^{18}$

In fully turbulent flow, $d\left\langle\sigma_{y}^{2}\right\rangle / d x$ is expected to be independent of $\operatorname{Re}_{d}$ at all $x$. This implies that $\left\langle\sigma_{y}^{2}\right\rangle(x)$ is also $\operatorname{Re}_{d}$-independent at all $x$ in this flow regime, given the same initial distribution of the solute $\left[\left\langle\sigma_{y}^{2}(x=0)\right\rangle=0\right]$. Indeed, $\left\langle\sigma_{y}^{2}\right\rangle$ was observed ${ }^{16}$ to be $\operatorname{Re}_{d}$-independent above $\operatorname{Re}_{d} \approx 200$ at selected $\phi(=0.091$ and 0.20$)$ and $x$. In contrast, $\left\langle\sigma_{y}^{2}\right\rangle$ was observed ${ }^{16}$ to decrease as $\operatorname{Re}_{d}$ decreased below $\mathrm{Re}_{d} \approx 100$ at these $\phi$ and $x$. (These thresholds are identified in terms of a different Reynolds number in Ref. 16; here, they are expressed in terms of $\operatorname{Re}_{d}$ for consistency with the rest of this paper.) Recall that for $\phi>0.19$, asymptotic (long-distance) $d\left\langle\sigma_{y}^{2}\right\rangle / d x$ is not expected to depend strongly on $\mathrm{Re}_{d}$. Therefore, the observed $\operatorname{Re}_{d}$ dependence of $\left\langle\sigma_{y}^{2}\right\rangle$ at $\operatorname{Re}_{d} \leq 100$ must arise entirely from a $\mathrm{Re}_{d}$-dependent pre-asymptotic (shortdistance) dispersion.

The objective of the present study is to use laboratory observations to evaluate two conjectures for lateral dispersion in dense arrays: (i) asymptotic $d\left\langle\sigma_{y}^{2}\right\rangle / d x$ exhibits only a weak dependence on $\mathrm{Re}_{d}$ and (ii) the advective distance required for the solute to achieve this asymptotic behavior is strongly dependent on $\mathrm{Re}_{d}$ prior to the onset of full turbulence due to the $\operatorname{Re}_{d}$ dependence of pore-scale mixing. The latter conjecture is explained in detail in Sec. III. To the authors' knowledge, experimental measurements of lateral dispersion at transitional $\operatorname{Re}_{d}$ within homogeneous random cylinder arrays are not currently available in literature. Shavit and Brandon ${ }^{19}$ reported laboratory measurements in a $\phi=0.035$ array, but illustrations of their array suggest that the cylinder distribution was in fact not random. More recently, Serra et al. ${ }^{20}$ measured the lateral dispersion of solute released at the upstream edge of a random array of $\phi=0.10$, 0.20 , and 0.35 at $\operatorname{Re}_{d}=11-120$. Their estimates of $K_{y y} /(\langle\bar{u}\rangle d)$ are independent of $\operatorname{Re}_{d}$ within experimental uncertainty over $32<\operatorname{Re}_{d}<120$, which is consistent with conjecture (i) above. However, the $\phi$ dependence of their reported values disagrees with that reported in Ref. 4 (Fig. 2). Specifically, $K_{y y} /(\langle\bar{u}\rangle d)$ at $\phi=0.10$ and at $\phi=0.20$ were the same within experimental uncertainty, and $K_{y y} /(\langle\bar{u}\rangle d)$ was the smallest at $\phi=0.35$ in the experiments of Serra et al. ${ }^{20}$ This disagreement may be due to their ${ }^{20}$ flow not being fully developed at the solute source or measurements being collected at very short distances $(x / d=8-26)$. Although Serra et $a l .{ }^{20}$ reported that their estimates of $K_{y y}$ did not vary with $x$ within this range, comparison with experiments reported in the present paper suggests that they were sampling in the pre-asymptotic regime (Sec. III C).

In this study, a set of laser-induced fluorescence (LIF) experiments were performed in arrays of $\phi=0.20$ and 0.35 at selected $\mathrm{Re}_{d}$ in the range of $\mathrm{Re}_{d}=48-120$. Visualizations of pore-scale mixing are presented, and the observed $\mathrm{Re}_{d}$ dependence is discussed qualitatively (Sec. III A). Timeaveraged concentration profiles are discussed in Secs. III B and III $\mathrm{C}$, in terms of their deviation from a Gaussian distribution, their variance, and the evolution of these two parameters with $x$. The growth rate of the variance is also compared to the corresponding $K_{y y} /(\langle\bar{u}\rangle d)$ in fully turbulent flow presented in Ref. 4, and pre-asymptotic and asymptotic regimes are identified. 
TABLE I. Relevant parameters for previous (Ref. 4) and present experiments considered in this paper. $d /\left\langle s_{n}\right\rangle_{A}$ is predicted by Eq. (A16) in Ref. 4. $x_{c} / d$ is predicted by Eq. (9), with $\left\langle\sqrt{k}_{t} /\langle\bar{u}\rangle\right\rangle$ as predicted for fully turbulent flow by Eq. (4.1) in Ref. 4, and $l_{t} / d$ as predicted by Eq. (10). $\langle\bar{u}\rangle$ was approximated by its cross-sectional average. $n$ is the total number of LIF experiments at all $x$ for $\left(\phi, \operatorname{Re}_{d}\right)=(0.35,300-390)$ and only at $x$ for which ensemble-averaged values are derived for all other $\left(\phi, \operatorname{Re}_{d}\right)$. Values for $\operatorname{Re}_{d}$, $\langle\bar{u}\rangle$, and $\langle\bar{H}\rangle$ represent the range across these replicate runs at the solute source.

\begin{tabular}{|c|c|c|c|c|c|c|}
\hline$\phi$ & $d /\left\langle s_{n}\right\rangle_{A}$ & $\begin{array}{c}x_{c} / d \\
\text { (fully turbulent) }\end{array}$ & $\operatorname{Re}_{d}$ & $\begin{array}{c}\langle\bar{u}\rangle \\
(\mathrm{cm} / \mathrm{s})\end{array}$ & $\begin{array}{l}\langle\bar{H}\rangle \\
(\mathrm{cm})\end{array}$ & $n$ \\
\hline \multirow[t]{4}{*}{0.20} & 2.70 & $\sim 5.9$ & $58-61$ & $0.82-1.4$ & $12.7-16.8$ & 57 \\
\hline & & & $84-89$ & $1.3-2.0$ & $13.5-16.9$ & 62 \\
\hline & & & $110-120$ & $1.6-2.5$ & $12.8-16.5$ & 39 \\
\hline & & & $310-340$ & $5.0-6.5$ & $11.8-15.1$ & $23^{\mathrm{a}}$ \\
\hline \multirow[t]{4}{*}{0.35} & 5.95 & $\sim 11$ & $48-51$ & $0.93-1.1$ & $12.8-14.8$ & 22 \\
\hline & & & $77-82$ & $1.4-1.8$ & $13.5-15.0$ & 30 \\
\hline & & & 97-100 & $1.4-2.2$ & $13.5-15.3$ & 31 \\
\hline & & & $300-390$ & $4.5-5.6$ & $13.3-20.3$ & $17^{\mathrm{a}}$ \\
\hline
\end{tabular}

${ }^{\mathrm{a}}$ These experiments were reported in Ref. 4.

\section{MATERIALS AND METHODS}

\section{A. Flume and cylinder array configuration}

Laboratory experiments were conducted in a recirculating Plexiglas flume with a $(x \times y)=284 \mathrm{~cm} \times 40 \mathrm{~cm}$ test section. The volumetric flow rate was measured with an in-line flow meter; its temporal variations ranged in magnitude from $1 \%$ to $4 \%$ in the present experiments. Flow is approximately uniform within an emergent array. ${ }^{4,12}$ Therefore, the temporally and spatially averaged interstitial velocity $\langle\bar{u}\rangle$ is approximated by its cross-sectional average, which was calculated as the temporal average of the volumetric flow rate divided by the width of the test section, the temporally and spatially averaged water depth $(\langle\bar{H}\rangle)$, and $1-\phi$. Note that the mean water depth decreases along the length of the array to balance the net drag exerted on the flow. ${ }^{21}$ The maximum decrease in mean water depth between the solute source and the LIF measurement location was $17 \%$ of the depth at the solute source. For consistency, $\langle\bar{u}\rangle$, and thus $\operatorname{Re}_{d}$, for a given experiment will be defined at the solute source in this paper. The range of experimental conditions examined is summarized in Table I.

Cylindrical maple dowels of diameter $d=0.64 \mathrm{~cm}$ (Saunders Brothers, Inc.) were inserted into perforated polyvinyl chloride sheets of either $20 \%$ or $35 \%$ hole fraction to create $280 \mathrm{~cm}$-long arrays of two densities: $\phi=0.20$ and 0.35 . These custom-made sheets were manufactured previously ${ }^{4}$ by generating uniformly distributed random coordinates for the hole centers until the desired number of nonoverlapping holes was assigned; nonoverlapping holes were defined to not have any other hole center fall within a $2 d \times 2 d$ square around its center. The $\phi=0.35$ array was created by filling all of the holes in the $35 \%$ hole fraction sheets. Different $\phi=0.20$ arrays were created by selecting different combinations of the $20 \%$ and $35 \%$ hole fraction sheets. In doing so, more array realizations could be included in the
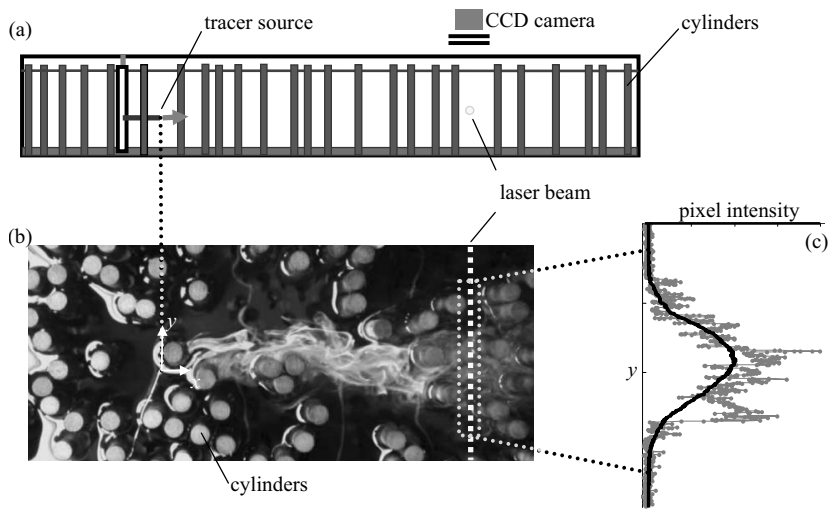

FIG. 3. Side (a) and plan (b) views of the test section of the experimental setup. Mean flow $\langle\bar{u}\rangle$ is left to right. Pixel intensity was extracted along a lateral transect from each image [gray dots in (c)] as a measure of the instantaneous concentration profile. The thick line in (c) is the temporally averaged profile.

ensemble average. Where $35 \%$ hole fraction sheets were used to create a $\phi=0.20$ array, the holes that were filled were selected using MATLAB's random number generator. The cylinders are perpendicular to the horizontal bed of the test section.

\section{B. LIF experiments}

LIF was used to measure the lateral concentration distribution at $\left(x, \mathrm{Re}_{d}\right)$ selected systematically between $\mathrm{Re}_{d}=48$ and 120 and $x=38$ and $234 \mathrm{~cm}$ ( $x=0$ is the solute source). The procedure was essentially the same as that described in Ref. 4, so only key steps are presented here. Dilute rhodamine WT was injected continuously with a syringe pump (Orion Sage ${ }^{\mathrm{TM}}$ M362) from a needle embedded within the array. The rate of injection was matched visually to the local flow. A single horizontal beam of argon ion laser (Coherent INNOVA $^{R} 70$ and $70 \mathrm{C}$ ion laser) passed laterally through the flume at a single streamwise position, $x$, downstream of the solute source (Fig. 3). A charge-coupled device camera (Sony XCD-X710) recorded the line of fluoresced solute from above the flume in a sequence of $1024 \times 48$ bitmap images. 530 and $515 \mathrm{~nm}$ long-pass filters (Midwest Optical Systems, Inc.) were attached to the camera to filter out the laser beam. Preliminary measurements confirmed that the recorded fluorescence intensity was linearly proportional to rhodamine WT concentration; for simplicity, the former was used in all analyses. For each experiment, cylinders were removed to create a single $1.3-\mathrm{cm}$ gap in the array, which allowed the laser beam to pass through the entire width of the flume.

Instantaneous intensity profiles were extracted from the images, corrected for background and anomalous pixel intensities, and averaged over the duration of the experiment to yield a time-averaged intensity profile, $\overline{I(y, t)}$. The duration of the experiments in $\phi=0.20$ ranged from 46 to $213 \mathrm{~s}$ (4.5-18.9 frames/s). In $\phi=0.35$, images were collected over $94 \mathrm{~s} \mathrm{(10.1-10.7} \mathrm{frames/s)} \mathrm{in} \mathrm{all} \mathrm{but} \mathrm{six} \mathrm{runs;} \mathrm{the} \mathrm{dura-}$ tion of the other six runs ranged from 80 to $112 \mathrm{~s}(9.0-12.5$ frames/s). The high frame rate at $\phi=0.20$ arose because one of the two computers used was able to record images at a 
faster frame rate. The time-averaged profile was further corrected for noise and background. Two quantitative parameters were considered in describing $\overline{I(y, t)}$. First, its variance was calculated as

$$
\sigma_{y}^{2}(x)=\frac{m_{2}(x)}{m_{0}(x)}-\left[\frac{m_{1}(x)}{m_{0}(x)}\right]^{2},
$$

where $m_{j}(x)$ is the $j$ th moment,

$$
m_{j}(x)=\int_{\kappa_{2}}^{\kappa_{1}} y^{j} \overline{I(y, t)} d y .
$$

$y=0$ is defined at the lateral position of the solute source. The zeroth, first, and second moments and the corresponding $\sigma_{y}^{2}$ were calculated by setting the limits of integration in Eq. (6), $\kappa_{1,2}$, at the two edges of the images. Next, $\kappa_{1,2}$ were redefined as $\kappa_{1,2}=\left(m_{1} / m_{0}\right) \pm 4 \sigma_{y}$ and the calculation was repeated. These limits were applied to prevent small fluctuations at large distances from the center of mass from unrealistically increasing the variance estimate. Second, the deviation of $\overline{I(y, t)}$ from a Gaussian distribution was parameterized by

$$
C_{m s}=\frac{1}{\kappa_{1}-\kappa_{2}} \int_{\kappa_{2}}^{\kappa_{1}} \frac{\left\{\overline{I(y, t)} / m_{0}-I_{G}(y)\right\}^{2}}{I_{G}(y)} d y,
$$

where $\kappa_{1,2}=\left(m_{1} / m_{0}\right) \pm 3 \sigma_{y}$ and $I_{G}(y)$ is a Gaussian distribution with unit total mass and with the same center of mass $\left(m_{1} / m_{0}\right)$ and $\sigma_{y}^{2}$ as $\overline{I(y, t)}$.

\section{EXPERIMENTAL RESULTS AND DISCUSSION}

By analogy with turbulent diffusion, dispersion in a homogeneous array is expected to become Fickian once the plume becomes well-mixed at, and significantly larger than, the spatial scales of all contributing mechanisms. ${ }^{9,11,12}$ In steady laminar flows and in turbulent flows through dense random arrays, the mechanisms that contribute to (macroscopic) lateral dispersion are molecular diffusion, turbulent diffusion, and dispersion associated with the tortuous flow path. Turbulent eddies are constrained by the local cylinder spacing, and thus the largest eddies scale with $\left\langle s_{n}\right\rangle_{A}$ in dense arrays. ${ }^{4}$ Dispersion associated with the tortuous flow path can be modeled as a series of independent, discrete lateral deflections that fluid particles undergo as they flow around cylinders; each deflection is expected to scale with $d^{22,23}$ Accordingly, asymptotic dispersion is expected once $\left\langle\sigma_{y}^{2}\right\rangle \gg d^{2},\left\langle s_{n}\right\rangle_{A}^{2}$.

The initial (small $x$ ) growth of the ensemble-averaged variance of the plumes may be approximated by $\left\langle\sigma_{y}^{2}\right\rangle=2 D_{\text {pore }} t=2 D_{\text {pore }} x /\langle\bar{u}\rangle$, where the diffusion coefficient $D_{\text {pore }}$ characterizes pore-scale mixing. Then, the mean streamwise distance that the solute is advected before achieving asymptotic behavior, $x_{c}$, is expected to scale as

$$
\frac{x_{c}}{d} \sim\left(\frac{\max \left\{d,\left\langle s_{n}\right\rangle_{A}\right\}}{d}\right)^{2} \frac{\langle\bar{u}\rangle d}{D_{\text {pore }}} .
$$

In steady laminar flow, $D_{\text {pore }}$ is the molecular diffusion coefficient $D_{m}$, and $x_{c} / d$ is linearly proportional to the Peclet number $\mathrm{Pe} \equiv\langle\bar{u}\rangle d / D_{m}$. This Pe dependence has been ob-

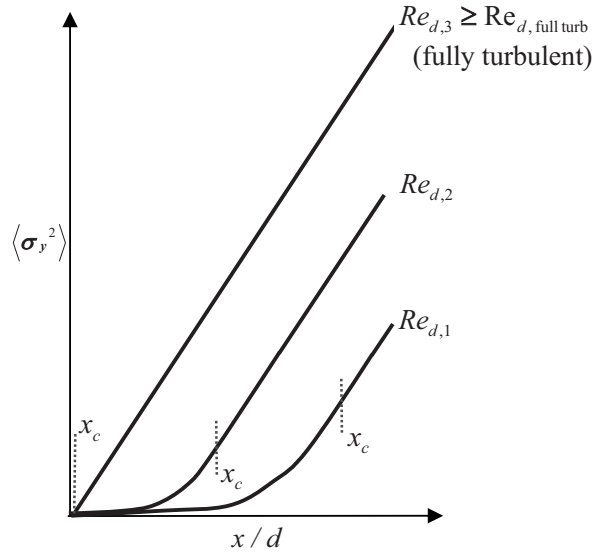

FIG. 4. Anticipated evolution of the variance of the lateral concentration profile with distance, and its dependence on $\operatorname{Re}_{d}$ in unsteady laminar and turbulent flows in dense arrays. The distance required to reach the asymptotic limit at a given $\mathrm{Re}_{d}, x_{c}$ (dotted), is described by Eq. (8). $\operatorname{Re}_{d, 3} \geq \operatorname{Re}_{d, \text { full turb }}>\operatorname{Re}_{d, 2}>\operatorname{Re}_{d, 1}$, where $\operatorname{Re}_{d, \text { full turb }}(\phi)$ denotes the $\operatorname{Re}_{d}$ at which flow becomes fully turbulent. Not to scale.

served in simulations of different types of steady laminar obstructed flows, e.g., in packed beds of spheres, ${ }^{24,25}$ in periodic cylinder arrays, ${ }^{3}$ and in a lattice network. ${ }^{26}$ In contrast, in turbulent flow, turbulent mixing is the dominant mechanism for pore-scale mixing. Then, $D_{\text {pore }} \sim\left\langle\sqrt{k_{t}}\right\rangle l_{t}$, where $k_{t}$ is the turbulent kinetic energy per unit mass and $l_{t}$ is the integral length scale of turbulence. Recall that $d>\left\langle s_{n}\right\rangle_{A}$ in the arrays considered in the present paper (Sec. I; Table I). Under these conditions, Eq. (8) yields

$$
\frac{x_{c}}{d} \sim\left(\left\langle\frac{\sqrt{k_{t}}}{\langle\bar{u}\rangle}\right\rangle \frac{l_{t}}{d}\right)^{-1} \text {. }
$$

It was previously ${ }^{4}$ shown that the integral length scale is accurately described by $l_{t} / d=\min \left\{1,\left\langle s_{n}\right\rangle_{A} / d\right\}$ in fully turbulent flow. Then, in the arrays considered in this paper,

$$
\frac{l_{t}}{d}=\frac{\left\langle s_{n}\right\rangle_{A}}{d},
$$

which decreases monotonically as $\phi$ increases. Further, Eq. (10) is expected to be valid in transitional turbulent flow as well, because the physical reasoning behind it, i.e., that the cylinder spacing limits the size of the largest turbulent eddies, involves only a purely geometric constraint. In contrast, mean turbulence intensity, $\left\langle{\sqrt{k_{t}}}_{t}\langle\bar{u}\rangle\right\rangle$, is expected to depend strongly on $\operatorname{Re}_{d}$. In fully turbulent flow, $\left\langle\sqrt{k_{t}} /\langle\bar{u}\rangle\right\rangle$ can be described by scale models in Ref. 4; at smaller $\operatorname{Re}_{d}$, values for $\left\langle\sqrt{k}_{t} /\langle\bar{u}\rangle\right\rangle$ are not available to our knowledge. Nevertheless, $\left\langle\sqrt{k_{t}} /\langle\bar{u}\rangle\right\rangle$ is expected to increase gradually and monotonically with $\mathrm{Re}_{d}$ between laminar and fully turbulent conditions. While we are not aware of any experimental verification of this expected $\mathrm{Re}_{d}$ dependence in packed beds or random cylinder arrays, it has been observed in ceramic foams $\mathrm{s}^{27}$ and in numerical simulations of a periodic cylinder array. ${ }^{28}$ With this assumption, Eq. (9) implies that in turbulent flow, $x_{c}$ decreases as $\mathrm{Re}_{d}$ increases until the flow becomes fully turbulent. The appropriate definition for $D_{\text {pore }}$ in unsteady laminar flow is not obvious. Nevertheless, $x_{c}$ is 


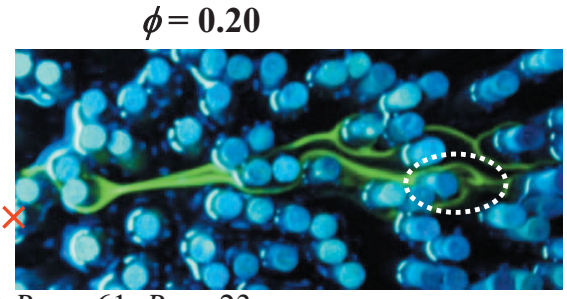

(a) $R e_{d}=61, R e_{\mathrm{s}}=23$

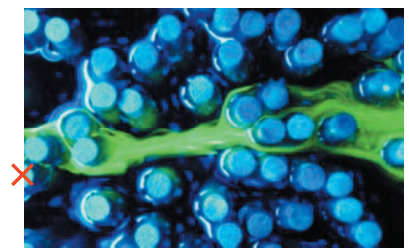

(b) $R e_{d}=94, R e_{\mathrm{s}}=35$

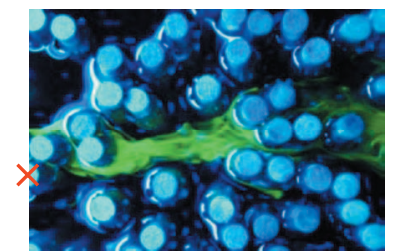

(c) $R e_{d}=120, R e_{\mathrm{s}}=46$

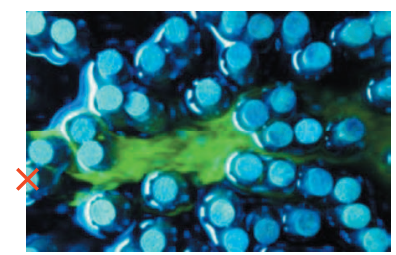

(d) $R e_{d}=190, R e_{\mathrm{s}}=70$

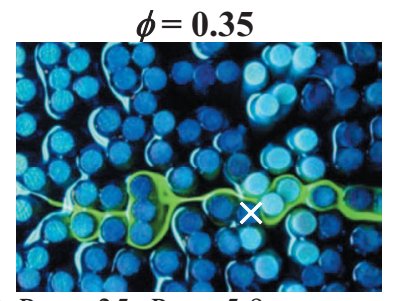

(e) $R e_{d}=35, R e_{\mathrm{s}}=5.9$

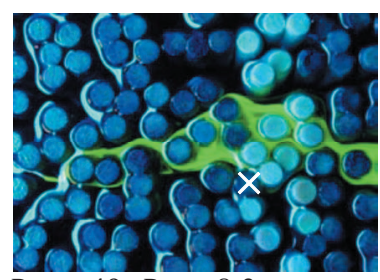

(f) $R e_{d}=49, R e_{\mathrm{s}}=8.3$

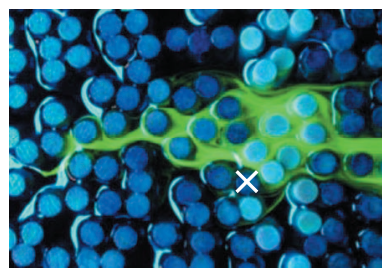

(g) $R e_{d}=81, R e_{\mathrm{s}}=14$

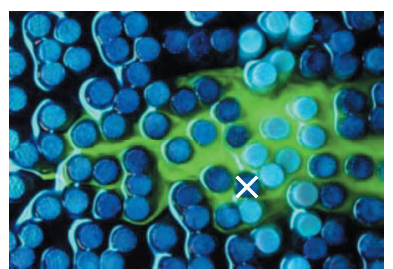

(h) $R e_{d}=180, R e_{\mathrm{s}}=30$
FIG. 5. (Color) Flow visualization by illuminating fluorescein with blue lighting (Current Inc.) in a $\phi=0.20$ array at $\mathrm{Re}_{d}=$ (a) 61, (b) 94, (c) 120, and (d) 190 and in a $\phi=0.35$ array at $\operatorname{Re}_{d}=$ (e) 35, (f) 49, (g) 81, and (h) 180. These values correspond to $\operatorname{Re}_{s}=$ (a) 23, (b) 35, (c) 46, (d) 70, (e) 5.9 , (f) 8.3 , (g) 14 , and (h) 30. Mean flow was from left to right. The fluorescein injection position was fixed over (a)-(d) and over (e)-(h). In (a)-(d), fluorescein was injected along the upstream edge of the cylinder marked with a $\times$; in $(\mathrm{e})-(\mathrm{h})$, the source is visible in the photo. In (e)-(h), the distance between the source and the cylinder marked with a white $X$ is $6.0 \mathrm{~cm}$. Vortex shedding can be observed downstream of one of the cylinders in (a) (dotted oval). expected to be continuous over all $\mathrm{Re}_{d}$, which requires that $x_{c}$ decrease as the flow transitions from the steady laminar flow regime to the turbulent flow regime.

In summary, dispersion in dense arrays $(\phi>0.19)$ is expected to exhibit three properties. First, dispersion at large $x$ is expected to be Fickian, i.e., $d\left\langle\sigma_{y}^{2}\right\rangle / d x \neq f(x)$, at all $\operatorname{Re}_{d}$ (Sec. I). Second, $d\left\langle\sigma_{y}^{2}\right\rangle / d x$ in this asymptotic limit is expected to be approximately the same for all $\operatorname{Re}_{d}$ (Sec. I). Third, the characteristic distance required to reach this limit, $x_{c}$, is expected to decrease as $\operatorname{Re}_{d}$ increases beyond steady laminar flow, until fully turbulent flow is achieved [Eq. (9)]. The simplest dependence of $\left\langle\sigma_{y}^{2}(x)\right\rangle$ on $x$ and $\operatorname{Re}_{d}$ that would satisfy these assumptions is depicted in Fig. 4 for a given $\phi$. Note that this schematic further assumes that $d\left\langle\sigma_{y}^{2}\right\rangle / d x$ at a given $\operatorname{Re}_{d}$ increases monotonically as it approaches its asymptotic (large $x$ ) limit. With this pre-asymptotic behavior, $\left\langle\sigma_{y}^{2}(x)\right\rangle$ at a given $x$ necessarily increases with $\mathrm{Re}_{d}$ until fully turbulent flow is reached for all $x$, consistent with previous ${ }^{16}$ observations (Fig. 4). It should be noted that in flows in which the lateral velocity autocorrelation function takes on negative values, $d\left\langle\sigma_{y}^{2}\right\rangle / d x$ will decrease with increasing $x$ at some $x<x_{c}$ prior to achieving its asymptotic limit, as highlighted in the classic paper by Taylor. ${ }^{29} \mathrm{~A}$ transient decrease in the dispersion coefficient has been observed in numerical simulations of randomly packed bed of spheres $^{25}$ and of lattice networks. ${ }^{26}$

\section{A. Pore-scale mixing and the approach to asymptotic dispersion}

Pore-scale mixing was visualized using fluorescein for both solid volume fractions $(\phi=0.20$ and 0.35$)$ in steady laminar flow and in unsteady flow. The instantaneous distribution of fluorescein immediately downstream of its injection point is depicted in Fig. 5. It should be noted that the LIF measurements, discussed in Secs. III B and III C, were collected farther downstream $(x \geq 59 d)$. Beginning at the lowest $\mathrm{Re}_{d}$, tracer streaklines remained stationary in time, indicating steady flow, everywhere in Figs. 5(a) and 5(e) except in the region immediately downstream of the cylinder marked with an oval in Fig. 5(a). Vortices shed from the marked cylinder, indicating that flow was unsteady in that region. This simultaneous occurrence of both steady and un- 

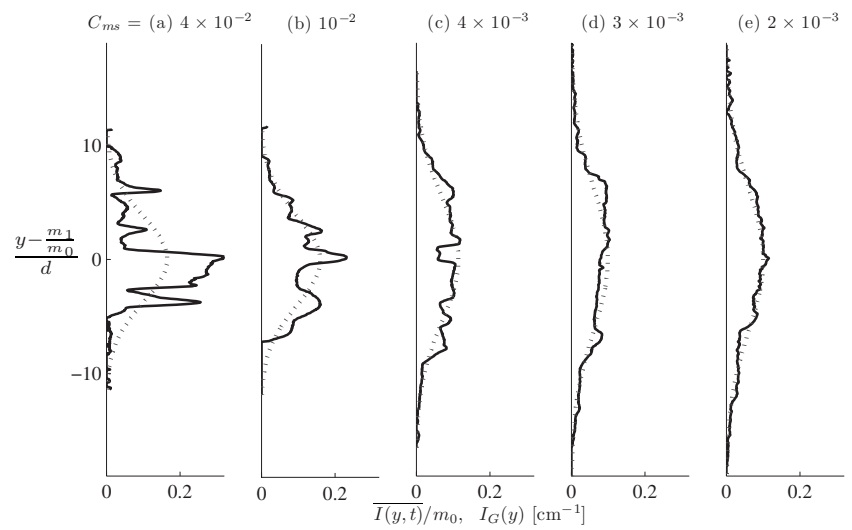

FIG. 6. $\overline{I(y, t)} / m_{0}\left(\mathrm{~cm}^{-1}\right)$ (solid) and the corresponding $I_{G}(y)$ [dotted; see Eq. (7)] for selected runs at $\left(\phi, \operatorname{Re}_{d}\right)=(0.35,97-100) . x \phi / d$ increases from left to right: (a) 21, (b) 42, (c) 58, (d) 87, and (e) 100. $C_{m s}$ values are also in $\left(\mathrm{cm}^{-1}\right)$. The growth of $\sigma_{y}^{2}$ with $x$ can be discerned from the width of the profiles, which are truncated at $y=m_{1} / m_{0} \pm 3 \sigma_{y}$.

steady flow at $\left(\phi, \operatorname{Re}_{d}\right)=(0.20,61)$ is attributed to the random distribution of the cylinders and the resulting spatial variations in the local velocity field.

In the absence of turbulence, the tracer is mixed with ambient fluid only through molecular diffusion, which is a very slow process: $D_{m} \approx 3 \times 10^{-6} \mathrm{~cm}^{2} / \mathrm{s}$ for rhodamine WT (estimated from Fig. 18.10 in Ref. 30) and $D_{m} \approx(3-5) \times 10^{-6} \mathrm{~cm}^{2} / \mathrm{s}$ for fluorescein. ${ }^{31-33}$ In the time taken for the tracer to advect to the right edge of the photo in Fig. 5(a) $(x \approx 22 d)$ and Fig. 5(e) $(x \approx 15 d)$, molecular diffusion can only have mixed the tracer over $O(0.01 d)$. Accordingly, thin, distinct filaments are observed in the photographs. In contrast, at $\operatorname{Re}_{d}>120$ in the $\phi=0.20$ array and at
$\mathrm{Re}_{d}=180$ in the $\phi=0.35$ array, the interface between the tracer and the ambient fluid was blurred, and the tracer was well mixed at the pore scale and distributed over distances larger than $d$ and $\left\langle s_{n}\right\rangle_{A}$ by the time it reached the right edge of the respective photos [Figs. 5(c), 5(d), and 5(h)]. The rapid pore-scale mixing and the absence of any apparent periodicity are indicative of turbulent flow, in which smallscale turbulence provides an additional, much faster mechanism for mixing within the pores. Recall that the tracer plume must be well mixed and much wider than $d$ and $\left\langle s_{n}\right\rangle_{A}$ for its dispersion to be asymptotic. Based on the observations above, we expect that beyond the steady laminar flow regime, the distance solute particles are advected before their dispersion becomes asymptotic, $x_{c}$, is smaller at higher $\mathrm{Re}_{d}$.

\section{B. Deviation of the time-averaged concentration profile from a Gaussian distribution}

Fickian dispersion of solute released from a point source results in a Gaussian concentration distribution. Therefore, the deviation of the time-averaged concentration profile, $\overline{I(y, t)}$, from a Gaussian distribution is a convenient measure of the proximity to Fickian behavior. For reference, selected $\overline{I(y, t)}$ are presented in Fig. 6. The time-averaged profiles gradually approach a Gaussian distribution as $x$ increases, and this trend is reflected in the corresponding reduction in the mean normalized squared deviation, $C_{m s}$ [Eq. (7)].

The ensemble-averaged $C_{m s}$ are presented in Fig. 7 as a function of $x \phi / d$, which is the expected number of cylinders in a $d \times x$ area (multiplied by $\pi / 4$ ). Two salient trends can be identified at each $\phi$. First, $\left\langle C_{m s}\right\rangle$ decreases as $x$ increases at each $\mathrm{Re}_{d}$; second, $\left\langle C_{m s}\right\rangle$ decreases as $\operatorname{Re}_{d}$ increases for all $x$
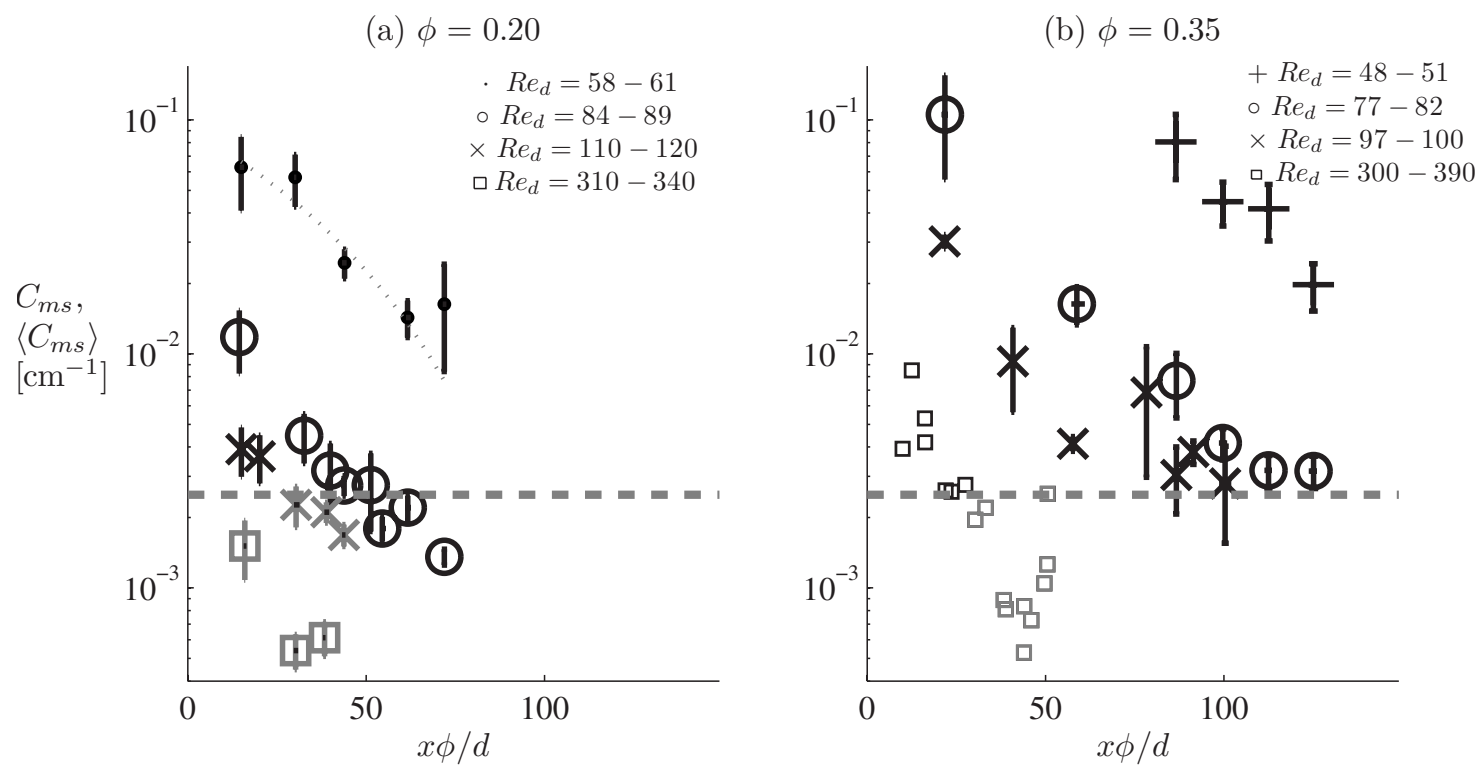

FIG. 7. Ensemble average of mean normalized squared deviation $C_{m s}\left(\mathrm{~cm}^{-1}\right)$ as defined by Eq. (7) for (a) $\phi=0.20$ at $\operatorname{Re}_{d}=58-61(\cdot), 84-89(\bigcirc), 110-120(\times)$, and 310-340 [( $\square)$ Ref. 4]; (b) $\phi=0.35$ at $\operatorname{Re}_{d}=48-51(+), 77-82(\bigcirc)$, and 97-100 (×). Each data point for these $\left(\phi, \operatorname{Re}_{d}\right)$ represents an average of four or more runs: horizontal bars indicate the range in $x$ and vertical bars indicate the standard error of the mean. (b) also includes individual $C_{m s}$ at $\left(\phi, \operatorname{Re}_{d}\right)=(0.35,300-390)\left[(\square)\right.$ Ref. 4]. Note that ensemble averages cannot be computed for this $\left(\phi, \operatorname{Re}_{d}\right)$ because replicate measurements were not collected. Data points in gray correspond to $\left(\phi, \operatorname{Re}_{d}, x\right)$ at which dispersion was observed to have occurred at the same rate as asymptotic dispersion in fully turbulent flow (Figs. 8 and 9). $\left\langle C_{m s}\right\rangle=2.5 \times 10^{-3} \mathrm{~cm}^{-1}$ (dashed) is the proposed empirical boundary between the pre-asymptotic and asymptotic dispersion regimes. Dotted line is provided only as a guide to the eye. 


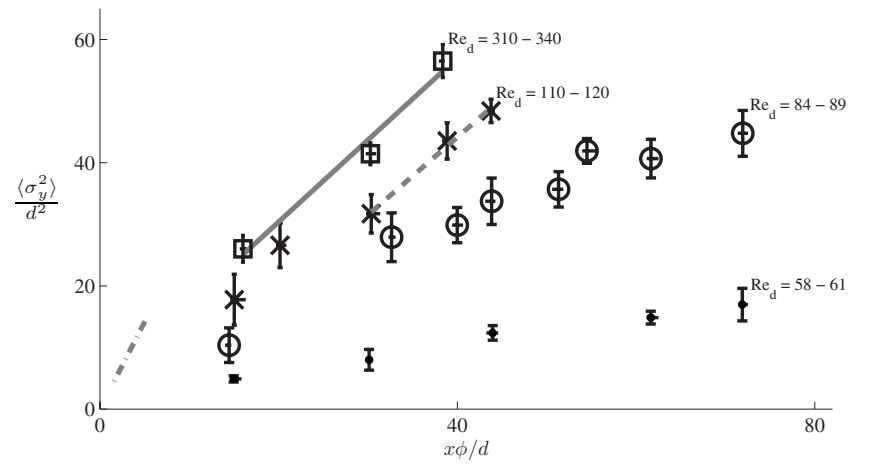

FIG. 8. Evolution of the ensemble-averaged variance with normalized distance at $\phi=0.20$. Markers same as Fig. 7(a). Solid line is the least-squares fit to $\left\langle\sigma_{y}^{2}\right\rangle(x)$ at $\operatorname{Re}_{d}=310-340: d\left\langle\sigma_{y}^{2}\right\rangle / d x=0.17 \pm 0.03 \mathrm{~cm} \quad\left(r^{2}=0.98, n=3\right)$. Dashed line is the least-squares fit to $\left\langle\sigma_{y}^{2}\right\rangle(x)$ at $\operatorname{Re}_{d}=110-120, x \phi / d>30$ : $d\left\langle\sigma_{y}^{2}\right\rangle / d x=0.16 \pm 0.01 \mathrm{~cm}\left(r^{2}=0.99, n=3\right)$. Also included is $\left\langle\sigma_{y}^{2}\right\rangle / x$ $=0.56 \mathrm{~cm}$ (dashed-dotted), as measured by Serra et al. (Ref. 20) at $37<\operatorname{Re}_{d} \leq 110$.

considered. The former trend is attributed to the plume having had more time to mix over the pore scale at larger $x$, and the latter trend is attributed to the enhancement of pore-scale mixing that results from the increase in turbulence intensity with increasing $\mathrm{Re}_{d}$. Note that the large reduction in $\left\langle C_{m s}\right\rangle$ between $\operatorname{Re}_{d}=58-61$ and $84-89$ at $\phi=0.20$ coincides with the transition from steady to unsteady flow captured in Figs. 5(a) and 5(b). A similar reduction occurred at $\phi=0.35$ between $\operatorname{Re}_{d}=48-51$ and 77-82. Note that the ranges in $\operatorname{Re}_{d}$ reflect unintended small variations across replicate runs, not measurement uncertainty (Table I).

At similar $x \phi / d$ and $\operatorname{Re}_{d},\left\langle C_{m s}\right\rangle$ was consistently larger at $\phi=0.35$ than at $\phi=0.20$ (Fig. 7). This trend implies that at larger $\phi$, a longer advective distance in terms of the number of cylinders per $d$-width is required for dispersion to become Fickian for the same $\operatorname{Re}_{d}$. This $\phi$ dependence is expected in fully turbulent flow, for which Eq. (9) predicts $x_{c} \phi / d \sim 4$ for $\phi=0.35$ and $\sim 1$ for $\phi=0.20$ (Table I). Unfortunately, the $\phi$ dependence of $x_{c} \phi / d$ at transitional $\operatorname{Re}_{d}$ cannot be predicted from Eq. (9) because measurements of $\left\langle\sqrt{k_{t}} /\langle\bar{u}\rangle\right\rangle$ are not available.

\section{Variance of the time-averaged concentration profile}

Measurements of the ensemble-averaged variance of time-averaged lateral concentration profiles $\left(\left\langle\sigma_{y}^{2}\right\rangle\right)$ are presented in Figs. 8 and 9 for $\phi=0.20$ and 0.35 , respectively. A subset of previous ${ }^{4}$ measurements at high $\mathrm{Re}_{d}$ is also included ( $\square$ ). As discussed in Sec. I, $\left\langle\sigma_{y}^{2}\right\rangle$ at a given $x$ becomes $\operatorname{Re}_{d}$ independent at $\operatorname{Re}_{d} \approx 200$ in a $\phi=0.20$ array. ${ }^{16}$ The high $\operatorname{Re}_{d}(=310-340)$ data for $\phi=0.20$ were selected from this regime, i.e., both $\left\langle\sigma_{y}^{2}\right\rangle$ and $d\left\langle\sigma_{y}^{2}\right\rangle / d x$ are independent of $\operatorname{Re}_{d}{ }^{4}$ At $\phi=0.35, \operatorname{Re}_{d}$-independent $\left\langle\sigma_{y}^{2}\right\rangle$ could not be confirmed under experimental conditions considered previously ${ }^{4,16}$ or in the present study, i.e., at $\operatorname{Re}_{d}<400$. Therefore, $\sigma_{y}^{2}(x)$ at $\left(\phi, \operatorname{Re}_{d}\right)=(0.35,300-390)$ is expected to be smaller than in fully turbulent flow for all $x$ (cf. Fig. 4). Unfortunately, the laboratory flume cannot accommodate $\operatorname{Re}_{d}>400$ at this $\phi .{ }^{4}$

Both $\left\langle\sigma_{y}^{2}(x)\right\rangle$ and $d\left\langle\sigma_{y}^{2}\right\rangle / d x$ decrease as $\operatorname{Re}_{d}$ decreases

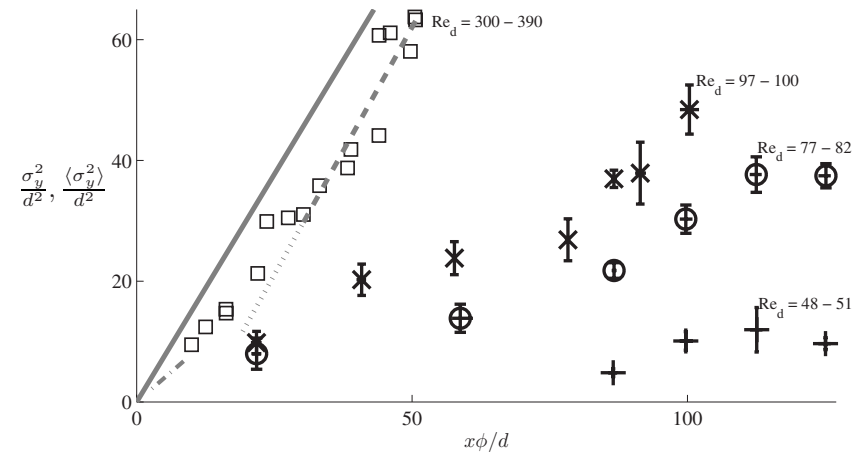

FIG. 9. Evolution of the ensemble-averaged variance with normalized distance at $\phi=0.35: \operatorname{Re}_{d}=48-51(+), 77-82(\bigcirc)$, and 97-100 $(\times)$. Individual $\sigma_{y}^{2}$ measurements from experiments at $\operatorname{Re}_{d}=300-390(\square)$ reported in Ref. 4 are also included. Each,$+ \bigcirc$, and $\times$ represents an average of four or more runs; horizontal bars indicate the range in $x$ and vertical bars indicate the standard error of the mean. Solid line depicts $d\left\langle\sigma_{y}^{2}\right\rangle / d x=0.34 \mathrm{~cm}$, as predicted for fully turbulent flow by Eq. (2.21) in Ref. 4, with scaling constants proposed therein. Dashed line is the least-squares fit to $\sigma_{y}^{2}(x)$ for $\operatorname{Re}_{d}$ $=300-390, x \phi / d>30: d \sigma_{y}^{2} / d x=0.36 \pm 0.05 \mathrm{~cm}\left(r^{2}=0.86, n=10\right)$. Dotted line is its extrapolation to smaller $x$, for reference. Also included is $\left\langle\sigma_{v}^{2}\right\rangle / x$ $=0.28 \mathrm{~cm}$ (dashed-dotted), as measured by Serra et al. (Ref. 20) at $46<\operatorname{Re}_{d} \leq 120$.

below $\operatorname{Re}_{d} \approx 100$ at both $\phi$ (Figs. 8 and 9). As depicted in Fig. 4, this $\operatorname{Re}_{d}$ dependence of $d\left\langle\sigma_{y}^{2}\right\rangle / d x$ at transitional $\operatorname{Re}_{d}$ is attributed to pre-asymptotic effects, i.e., the plume reached the measurement location before the solute particles experienced a sufficiently large subset of velocities in the array or, equivalently, before the solute particles dispersed over scales much larger than $d$ and $\left\langle s_{n}\right\rangle_{A}$ [Eq. (8)]. A qualitatively similar increase in $d\left\langle\sigma_{y}^{2}\right\rangle / d x$ with $\langle\bar{u}\rangle / \nu$ at a fixed $x$, and subsequent $\langle\bar{u}\rangle / \nu$ independence at high $\langle\bar{u}\rangle / \nu$, have been observed in ceramic foam. ${ }^{34,35}$

If the observed $\operatorname{Re}_{d}$ dependence of $d\left\langle\sigma_{y}^{2}\right\rangle / d x$ is indeed due to pre-asymptotic effects, then $d\left\langle\sigma_{y}^{2}(x)\right\rangle / d x$ is expected to approach its asymptotic value in fully turbulent flow at sufficiently large $x\left(=x_{c}\right)$ (Fig. 4). At $\left(\phi, \operatorname{Re}_{d}\right)$ $=(0.20,58-61)$, flow is steady and laminar [Fig. 5(a)], and the predicted $x_{c} \phi / d\left[\sim 3 \times 10^{4}\right.$, Eq. (8)] is two orders of magnitude larger than the length of the laboratory array $(x \phi / d=88)$. Accordingly, $d\left\langle\sigma_{y}^{2}\right\rangle / d x$ remains smaller than its asymptotic value at high $\operatorname{Re}_{d}(=310-340)$ for all $x$ considered [Fig. $8()$.$] . In contrast, at \left(\phi, \operatorname{Re}_{d}\right)=(0.20,110-120)$, $d\left\langle\sigma_{y}^{2}\right\rangle / d x$ is equal to its asymptotic value at high $\operatorname{Re}_{d}$ within uncertainty at $x \phi / d>30$, but deviates at $x \phi / d \leq 20$ [Fig. 8 $(\times)$ ]. This trend indicates that asymptotic dispersion is reached in the range $20<x_{c} \phi / d \leq 30$ at this $\left(\phi, \operatorname{Re}_{d}\right)$. This distance is larger than $x_{c} \phi / d \sim 1$, predicted by Eq. (9) for fully turbulent flow, presumably because the flow was not fully turbulent at this $\operatorname{Re}_{d}(=110-120)$ and the weaker turbulence intensity relative to fully turbulent flow extended $x_{c}$ (cf. Fig. 4).

It is convenient to relate numerical values of $C_{m s}$ to asymptotic/pre-asymptotic dispersion as interpreted directly from $d\left\langle\sigma_{y}^{2}\right\rangle / d x$. A threshold defined at $C_{m s}=2.5 \times 10^{-3} \mathrm{~cm}^{-1}$ accurately segregates $\left(\phi, \operatorname{Re}_{d}\right)$ $=(0.20,110-120)$ data in the asymptotic regime $(x \phi / d$ $>30)$ from those in the pre-asymptotic regime $(x \phi / d \leq 20)$, 
as determined above. With this threshold, $\left\langle C_{m s}\right\rangle$ for $\left(\phi, \operatorname{Re}_{d}\right)=(0.20,58-61)$ and $(0.20,310-340)$ are also correctly classified into the pre-asymptotic and asymptotic dispersion regimes, respectively [Fig. 7(a)].

This criterion can now be applied to classify the remaining measurements, for which the anticipated transition from pre-asymptotic to asymptotic (large $x$ ) dispersion cannot be easily identified by eye (Figs. 8 and 9). For example, the range of $x$ over which $d \sigma_{y}^{2} / d x$ is constant is not obvious for $\left(\phi, \mathrm{Re}_{d}\right)=(0.35,300-390)$. However, $\left\langle C_{m s}\right\rangle$ at $\left(\phi, \mathrm{Re}_{d}\right)$ $=(0.35,300-390)$ and $(0.20,84-89)$ fall below the threshold at $x \phi / d>30$ and $\geq 55$, respectively, implying that the asymptotic regime had been reached at these $x$. Least-squares fit to data in the former yields $d \sigma_{y}^{2} / d x=0.36 \pm 0.05 \mathrm{~cm}(\mathrm{co}-$ efficient of determination $r^{2}=0.86, n=10$ ) (Fig. 9, dashed line). The correlation is highly significant, confirming that dispersion is indeed Fickian at $x \phi / d>30$. Furthermore, the best-fit $d \sigma_{y}^{2} / d x$ is equal, within uncertainty, to that predicted for fully turbulent flow by Eq. (2.21) in Ref. 4, with scaling constants proposed therein (Fig. 9, solid line). This agreement supports the conjecture that asymptotic $d\left\langle\sigma_{y}^{2}\right\rangle / d x$ is independent of $\mathrm{Re}_{d}$. In contrast, a linear regression at $55 \leq x \phi / d \leq 72$ for $\left(\phi, \operatorname{Re}_{d}\right)=(0.20,84-89)$ does not yield a significant correlation $\left(r^{2}=0.56, n=3\right)$. Since $\left\langle\sigma_{y}^{2}\right\rangle(x)$ measurements are constant within standard error in this range, the weak correlation is most likely due to measurements not extending sufficiently far into the asymptotic regime. Finally, $\left\langle C_{m s}\right\rangle$ (or $C_{m s}$ ) corresponding to $\left(\phi, \operatorname{Re}_{d}, x\right)$ at which measured $d\left\langle\sigma_{y}^{2}\right\rangle / d x$ (or $d \sigma_{y}^{2} / d x$ ) was identified above to have occurred at the same rate as asymptotic dispersion in fully turbulent flow are plotted in gray in Fig. 7. The empirical boundary $\left\langle C_{m s}\right\rangle=2.5 \times 10^{-3} \mathrm{~cm}^{-1} \quad$ (dashed) accurately segregates the ensemble-averaged time-averaged concentration profiles in the asymptotic regime from those in the preasymptotic regime.

\section{CONCLUSIONS}

The lateral distribution of passive solute released continuously from a point source was measured at $x / d=59-370$ and $\operatorname{Re}_{d}=48-120$ in random cylinder arrays of solid volume fraction $\phi=0.20$ and 0.35 , and the results were compared to previous ${ }^{4}$ measurements at $\operatorname{Re}_{d}=300-390$. Previous ${ }^{4}$ predictions for fully turbulent flow imply that asymptotic (large $x$ ) lateral dispersion, $K_{y y} /(\langle\bar{u}\rangle d)$, will not exhibit a strong dependence on $\operatorname{Re}_{d}$ at these $\phi$. Measured $d\left\langle\sigma_{y}^{2}\right\rangle / d x$ reached asymptotic rates predicted ${ }^{4}$ for fully turbulent flow at $\left(\phi, \operatorname{Re}_{d}\right)=(0.20,110-120)$ and $(0.35,300-390)$ at large $x$, supporting this conjecture. At the same time, it is apparent from flow visualization that turbulence substantially enhances pore-scale mixing, which in turn accelerates the approach to asymptotic dispersion. This $\mathrm{Re}_{d}$ dependence explains why dispersion at lower $\operatorname{Re}_{d}(<100)$ did not reach the fully turbulent flow limit within the length and width of the laboratory array.

Further, results suggest that at the same $\mathrm{Re}_{d}$, the preasymptotic regime extends farther downstream at $\phi=0.35$, in terms of the number of cylinders spanned $(x \phi / d)$, than at $\phi=0.20$. Concentration profiles within the asymptotic disper- sion regime deviated from a Gaussian distribution by less than $\left\langle C_{m s}\right\rangle=2.5 \times 10^{-3} \mathrm{~cm}^{-1}$ in both previous ${ }^{4}$ and present experiments. Using this as an empirical criterion, we can evaluate from time-averaged profiles at a single streamwise location whether dispersion at that distance is pre-asymptotic or asymptotic. This method requires significantly less effort than obtaining measurements at multiple streamwise positions to evaluate $d\left\langle\sigma_{y}^{2}\right\rangle / d x$.

Returning to our original motivation for this study, let us now consider typical conditions in aquatic systems with vegetation. The above discussion suggests that solute released into dense vegetation in the field undergoes pre-asymptotic dispersion through a significant portion of the system. A rough extrapolation of our laboratory data suggests that at $\mathrm{Re}_{d} \leq 60$, solute introduced in dense $(\phi>0.19)$ arrays must flow through a distance larger than $x \phi / d=100$ before its dispersion becomes asymptotic (Fig. 7). This value corresponds to $x=30 \mathrm{~m}$, for example, in a random array with the same $d(=3 \mathrm{~cm})$ and $\phi(\approx 0.1)$ as measured by Lightbody et al. ${ }^{36}$ in a constructed wetland $\left(\operatorname{Re}_{d}=30-40\right)$. This is a non-negligible distance in many aquatic systems (e.g., constructed wetlands, ${ }^{37}$ mangrove swamps, ${ }^{38,39}$ and salt marshes ${ }^{40}$ ), where homogeneous vegetation conditions extend no more than about $200 \mathrm{~m}$ perpendicular to land, and in some systems $^{37,40}$ much less. In particular, constructed wetlands typically comprise multiple cells of variable dimensions, and the shortest cell may only extend 30-300 m (Refs. 36 and 41-43) streamwise.

\section{ACKNOWLEDGMENTS}

This material is based on work supported by the National Science Foundation under Grant No. EAR0309188. Any opinions, conclusions, or recommendations expressed in this material are those of the authors and do not necessarily reflect the views of the National Science Foundation. We thank the three anonymous reviewers for their comments.

${ }^{1}$ T. Masuoka, Y. Takatsu, and T. Inoue, "Chaotic behavior and transition to turbulence in porous media," Nanoscale Microscale Thermophys. Eng. 6, 347 (2002).

${ }^{2}$ Y. Takatsu and T. Masuoka, "Turbulent phenomena in flow through porous media," J. Porous Media 1, 243 (1998).

${ }^{3}$ R. C. Acharya, A. J. Valocchi, C. J. Werth, and T. W. Willingham, "Porescale simulation of dispersion and reaction along a transverse mixing zone in two-dimensional porous media," Water Resour. Res. 43, W10435, DOI: 10.1029/2007WR005969 (2007).

${ }^{4}$ Y. Tanino and H. M. Nepf, "Lateral dispersion in random cylinder arrays at high Reynolds number," J. Fluid Mech. 600, 339 (2008).

${ }^{5}$ J. H. Lienhard, "Synopsis of lift, drag, and vortex frequency data for rigid circular cylinders," Bulletin 300 (Washington State University, College of Engineering Research Division, Technical Extension Service, Washington, 1966).

${ }^{6}$ P. K. Kundu and I. M. Cohen, Fluid Mechanics, 3rd ed. (Elsevier Academic, San Diego, CA, 2004)

${ }^{7}$ J. H. Gerrard, "The wakes of cylindrical bluff bodies at low Reynolds number," Philos. Trans. R. Soc. London, Ser. A 288, 351 (1978).

${ }^{8}$ J. J. Finnigan, "Turbulent transport in flexible plant canopies," in The Forest-Atmosphere Interaction, edited by B. A. Hutchison and B. B. Hicks (Reidel, Dordrecht, Holland, 1985), pp. 443-480.

${ }^{9}$ D. L. Koch and J. F. Brady, "Dispersion in fixed beds," J. Fluid Mech. 154, 399 (1985).

${ }^{10}$ D. L. Koch, R. J. Hill, and A. S. Sangani, "Brinkman screening and the covariance of the fluid velocity in fixed beds," Phys. Fluids 10, 3035 (1998). 
${ }^{11} \mathrm{~S}$. Corrsin, "Limitations of gradient transport models in random walks and in turbulence," Adv. Geophys. 18A, 25 (1974).

${ }^{12}$ B. L. White and H. M. Nepf, "Scalar transport in random cylinder arrays at moderate Reynolds number," J. Fluid Mech. 487, 43 (2003).

${ }^{13}$ H. B. Fischer, E. J. List, R. C. Y. Koh, J. Imberger, and N. H. Brooks, Mixing in Inland and Coastal Waters (Academic, New York, 1979).

${ }^{14}$ P. C. Chatwin and P. J. Sullivan, "Measurements of concentration fluctuations in relative turbulent diffusion," J. Fluid Mech. 94, 83 (1979).

${ }^{15}$ M. Dentz, H. Kinzelbach, S. Attinger, and W. Kinzelbach, "Temporal behavior of a solute cloud in a heterogeneous porous medium 1. Point-like injection," Water Resour. Res. 36, 3591, DOI: 10.1029/2000WR900162 (2000).

${ }^{16}$ Y. Tanino and H. M. Nepf, "Experimental investigation of lateral dispersion in aquatic canopies," in Proceedings of the 32nd Congress of IAHR, edited by G. Di Silvio and S. Lanzoni (International Association of Hydraulic Engineering and Research, Venice, Italy, 2007).

${ }^{17}$ R. J. Hill, D. L. Koch, and A. J. Ladd, "Moderate-Reynolds-number flows in ordered and random arrays of spheres," J. Fluid Mech. 448, 243 (2001).

${ }^{18}$ H. S. Mickley, K. A. Smith, and E. I. Korchak, "Fluid flow in packed beds," Chem. Eng. Sci. 20, 237 (1965).

${ }^{19} \mathrm{U}$. Shavit and T. Brandon, "Dispersion within emergent vegetation using PIV and concentration measurements," Proceedings of the 4th International Symposium on Particle Image Velocimetry (Institute of Aerodynamics and Flow Technology, Gottingen, Germany, 2001).

${ }^{20}$ T. Serra, H. J. S. Fernando, and R. V. Rodriguez, "Effects of emergent vegetation on lateral diffusion in wetlands," Water Res. 38, 139 (2004).

${ }^{21}$ Y. Tanino and H. M. Nepf, "Laboratory investigation of mean drag in a random array of rigid, emergent cylinders," J. Hydraul. Eng. 134, 34 (2008).

${ }^{22}$ H. M. Nepf, "Drag, turbulence, and diffusion in flow through emergent vegetation," Water Resour. Res. 35, 479, DOI: 10.1029/1998WR900069 (1999).

${ }^{23}$ T. Masuoka and Y. Takatsu, "Turbulence model for flow through porous media," Int. J. Heat Mass Transfer 39, 2803 (1996).

${ }^{24}$ S. Stapf, K. J. Packer, R. G. Graham, J.-F. Thovert, and P. M. Adler, "Spatial correlations and dispersion for fluid transport through packed glass beads studied by pulsed field-gradient NMR," Phys. Rev. E 58, 6206 (1998).

${ }^{25}$ R. S. Maier, D. M. Kroll, R. S. Bernard, S. E. Howington, J. F. Peters, and H. T. Davis, "Pore-scale simulation of dispersion," Phys. Fluids 12, 2065 (2000).

${ }^{26} \mathrm{~B}$. Bijeljic and M. J. Blunt, "Pore-scale modeling of transverse dispersion in porous media," Water Resour. Res. 43, W12S11, DOI: 10.1029/ 2006WR005700 (2007).
${ }^{27}$ M. J. Hall and J. P. Hiatt, "Measurements of pore scale flows within and exiting ceramic foams," Exp. Fluids 20, 433 (1996).

${ }^{28}$ R. J. Hill and D. L. Koch, "Moderate-Reynolds-number flow in a wallbounded porous medium," J. Fluid Mech. 453, 315 (2002).

${ }^{29}$ G. I. Taylor, "Diffusion by continuous movements," Proc. London Math. Soc. 2-20, 196 (1922).

${ }^{30}$ R. P. Schwarzenbach, P. M. Gschwend, and D. M. Imboden, Environmental Organic Chemistry, 2nd ed. (Wiley, Hoboken, NJ, 2003).

${ }^{31}$ S. A. Rani, B. Pitts, and P. S. Stewart, "Rapid diffusion of fluorescent tracers into Staphylococcus epidermidis biofilms visualized by time lapse microscopy," Antimicrob. Agents Chemother. 49, 728 (2005).

${ }^{32}$ K. C. Hodges and V. K. La Mer, "Solvent effects on the quenching of the fluorescence of uranin by aniline," J. Am. Chem. Soc. 70, 722 (1948).

${ }^{33}$ Z. Petrasek and P. Schwille, "Precise measurement of diffusion coefficients using scanning fluorescence correlation spectroscopy," Biophys. J. 94, 1437 (2008)

${ }^{34}$ J. C. F. Pereira, I. Malico, T. C. Hayashi, and J. Raposo, "Experimental and numerical characterization of the transverse dispersion at the exit of a short ceramic foam inside a pipe," Int. J. Heat Mass Transfer 48, 1 (2005).

${ }^{35}$ C. L. Hackert, J. L. Ellzey, O. A. Ezekoye, and M. J. Hall, "Transverse dispersion at high Peclet numbers in short porous media," Exp. Fluids 21, 286 (1996)

${ }^{36}$ A. F. Lightbody, M. E. Avener, and H. M. Nepf, "Observations of shortcircuiting flow paths within a free-surface wetland in Augusta, Georgia, U.S.A,” Limnol. Oceanogr. 53, 1040 (2008).

${ }^{37}$ R. H. Kadlec, "Detention and mixing in free water wetlands," Ecol. Eng. 3, 345 (1994).

${ }^{38}$ K. Furukawa, E. Wolanski, and H. Mueller, "Currents and sediment transport in mangrove forests," Estuarine Coastal Shelf Sci. 44, 301 (1997).

${ }^{39}$ D. Kobashi and Y. Mazda, "Tidal flow in riverine-type mangroves," Wetl. Ecol. Manag. 13, 615 (2005).

${ }^{40} \mathrm{U}$. Neumeier and C. L. Amos, "The influence of vegetation on turbulence and flow velocities in European salt-marshes," Sedimentology 53, 259 (2006).

${ }^{41}$ S. H. Keefe, L. B. Barber, R. L. Runkel, J. N. Ryan, D. M. McKnight, and R. D. Wass, "Conservative and reactive solute transport in constructed wetlands," Water Resour. Res. 40, W01201, DOI: 10.1029/ 2003WR002130 (2004).

${ }^{42}$ D. L. Hey, A. L. Kenimer, and K. R. Barrett, "Water quality improvement by four experimental wetlands," Ecol. Eng. 3, 381 (1994).

${ }^{43}$ C. J. Martinez and W. R. Wise, "Hydraulic analysis of Orlando Easterly Wetland," J. Environ. Eng. 129, 553 (2003). 